\title{
Evaluation of the pH-sensitive swelling of a hydrogel by means of a plasmonic sensor substrate
}

\author{
Christoph Kroh ${ }^{1,2}$, Roland Wuchrer ${ }^{2}$, Margarita Günther ${ }^{1}$, Thomas Härtling ${ }^{1,2}$, and Gerald Gerlach ${ }^{1}$ \\ ${ }^{1}$ Technische Universität Dresden, Institute of Solid State Electronics, \\ Mommsenstraße 15, 01069, Dresden, Germany \\ ${ }^{2}$ Fraunhofer Institute for Ceramic Technologies and Systems IKTS, \\ Maria-Reiche-Straße 2, 01109, Dresden, Germany \\ Correspondence: Christoph Kroh (christoph.kroh@tu-dresden.de)
}

Received: 8 September 2017 - Revised: 12 December 2017 - Accepted: 18 December 2017 - Published: 6 February 2018

\begin{abstract}
The inline monitoring of parameters in aqueous liquids is facing an increasing demand in many different application areas. Hydrogels with $\mathrm{pH}$-induced swelling and deswelling behavior offer a means to measure $\mathrm{pH}$ in such liquids. Here we investigate the optical interrogation of a $\mathrm{pH}$-sensitive hydrogel which can be applied in the physiological $\mathrm{pH}$ range. For this, a nanostructured gold substrate supporting surface plasmon oscillations is coated with a HPMA/DMAEMA/TEGDMA/EG hydrogel. The gel swells in the $\mathrm{pH}$ range under investigation (here 4.5 to 6.5 ), and the resulting refractive index changes subsequently lead to a spectral shift of the plasmon resonance of the gold nanostructure. The spectral resonance position is determined from optical transmittance spectra of the sensor substrates, and the initial results for our hydrogel reported here indicate a nearly linear dependence between the swelling state and the plasmon resonance wavelength.
\end{abstract}

\section{Introduction}

Efficient and low-cost sensors for the detection of chemical parameters in liquids (e.g., $\mathrm{pH}$ ) are facing an ever-increasing demand in many different application fields such as medical care, biotechnology, or industrial process monitoring. The measurement methods currently in use are typically based on electrical sensors, which require complex calibration (Wang et al., 2002; Wilhelm et al., 1991). An alternative approach for the measurement of $\mathrm{pH}$ is based on the use of $\mathrm{pH}$-responsive hydrogels. The latter are hydrophilic, nonsoluble, crosslinked polymers, which show an increase or decrease of volume as response to $\mathrm{pH}$ changes in their environment. Thus, interrogating the swelling-deswelling state of the hydrogel allows the $\mathrm{pH}$ to be deduced. The volume change can be detected, for instance, by means of a pressure sensor (Günther et al., 2013, 2014; Wang and Ward, 1993), which demands rather thick hydrogel sensor layers ranging from 300 to $500 \mu \mathrm{m}$ (Gerlach et al., 2004). Due to the dependence between layer thickness and response behavior (Tanaka and Fillmore, 1979), the equilibrium state of the hydrogels in these systems is typically reached only af- ter more than 30 min (Günther et al., 2013, 2014). One way to improve the response time of hydrogel-based sensors is to interrogate the $\mathrm{pH}$-induced swelling state optically, as this allows very thin layers to be used. To this end we bring a $\mathrm{pH}-$ sensitive hydrogel into contact with a nanostructured metal surface, which supports surface plasmon oscillations. The plasmon oscillation is sensitive to changes in the refractive index which occur during swelling-deswelling of the hydrogel, which, in turn, reflects in spectral changes in the transmittance spectrum of the metal nanostructure (Piliarik et al., 2012; Yang et al., 2015; Zalyubovskiy et al., 2012). The refractive index sensitivity is confined to the very surface of the nanostructure so that rather thin hydrogel layers can be used as $\mathrm{pH}$-sensitive material, which will consequently lead to faster response times.

The combination of a nanostructured metal surface and a $\mathrm{pH}$-sensitive hydrogel was reported previously in the literature (Mack et al., 2007; Jiang et al., 2009; Tokarev et al., 2008; Yao et al., 2010). However, it is still a challenge to use hydrogels in sensor systems with a durability of 12 weeks, which is a typical timescale for applications in the 


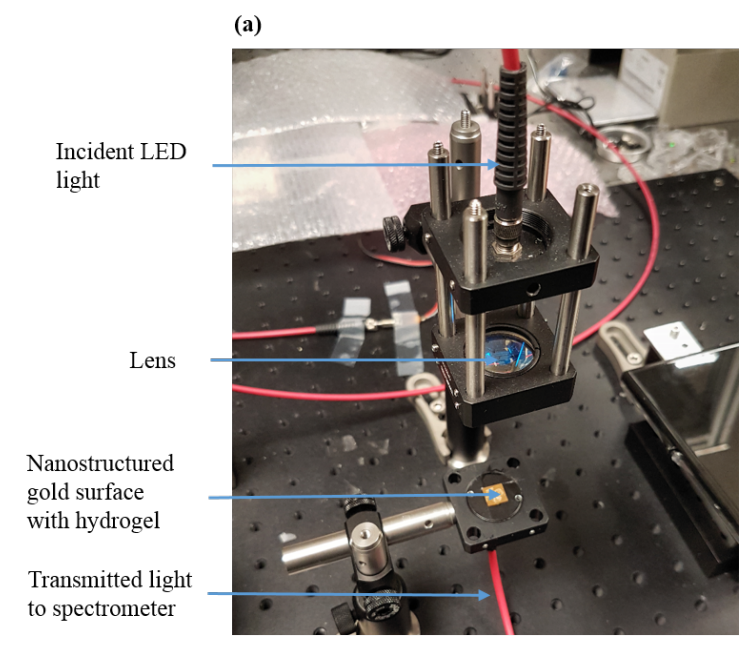

(b)

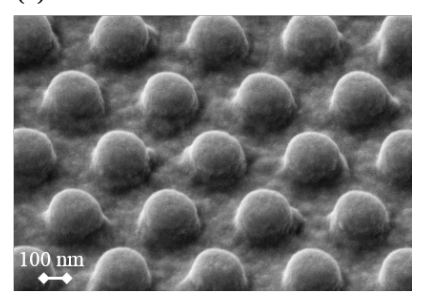

Figure 1. (a) Setup for analyzing the transmittance spectrum of the sensor substrates and (b) REM micrograph of the nanostructured gold surface.

medical and biotechnological sector. The overall aim of this work, therefore, is a robust hydrogel-based sensor for the inline measurement of $\mathrm{pH}$ with an operational lifetime of more than 10 days. As the first step, we report here on initial results on the optical detection of different swelling states of our $\mathrm{pH}$-sensitive hydrogel on a nanostructured gold surface. As we also envision implantable sensors for biomedical applications, a hydrogel composition is investigated, which can be used in the physiological pH range (Schulz et al., 2010). The combination of this specific hydrogel and a nanostructured plasmonic sensor substrate is studied for the first time, which provides data on the interrogation of physiologically applicable gels with optical methods. The initial results reported here show a nearly linear dependence between the swelling state and the plasmon resonance wavelength of the gold structure between $\mathrm{pH} 4.5$ and 6.5.

\section{Sensor setup}

The sensor consists of an optically active nanostructured gold substrate and a $\mathrm{pH}$-sensitive hydrogel layer for the analytespecific detection of refractive index changes. Gold nanostructures as used here are well known to support localized surface plasmon polaritons, which induce a refractiveindex-sensitive optical transmittance behavior (Kreibig et al., 1995). Such structures can therefore be used as signal transducers for the hydrogels. The optical interrogation of the sensor substrate is accomplished with a simple home-built transmittance evaluation system. The substrates are placed on an optical holder and are irradiated with a LED (875 nm central wavelength) in the spectral window between 750 and $1000 \mathrm{~nm}$. The light transmitted through the sensor substrate is collected with an optical fiber and directed to a spectrometer (iHR550, Horiba Jobin Yvon GmbH, Unterhaching, Ger- many). The entire setup and a REM micrograph of the nanostructured surface are depicted in Fig. 1.

The sensor signal originates from the superposition of the incident LED light spectrum and the extinction spectrum of the sensor substrate. From this superposition, a transmittance spectrum with a characteristic dip around $870 \mathrm{~nm}$ results. The dip, which represents the plasmon resonance wavelength, shifts upon refractive index changes occurring during the $\mathrm{pH}$ induced swelling of the hydrogel.

\section{Experimental}

\section{1 pH-sensitive hydrogel}

After fabrication and functionalization (see Sect. 3.2 and 3.3), the nanostructured gold sensor substrate is coated with a $\mathrm{pH}$-sensitive hydrogel layer (layer thickness around $30 \mu \mathrm{m})$ and purged with an ethanol-water solution for $24 \mathrm{~h}$. The gel consists of the monomers hydroxypropyl methacrylate (HPMA), 2-(dimethylamino)ethyl methacrylate (DMAEMA), tetraethylene glycol dimethacrylate (TEGDMA), and ethylene glycol (EG). HPMA is the monomer responsible for the bonding on organosilicon compounds. DMAEMA, as a co-monomer, contains the $\mathrm{pH}$-sensitive tertiary amines, TEGDMA acts as a crosslinker, and EG is used as a solvent. The HPMA/DMAEMA/TEGDMA/EG solution is produced according to Schmidt et al. (2016) in a molar ratio of $70 / 30 / 1 / 20$ and stirred for $15 \mathrm{~min}$ under a nitrogen atmosphere. After adding the photoinitiator 2-hydroxy-4'-(2hydroxyethoxy)-2-methylpropiophenone and stirring again for 15 min under a nitrogen atmosphere, the pregel is applied to the functionalized sensor substrates and distributed via spin-coating. Photopolymerization is achieved using an ultraviolet lamp for $5 \mathrm{~min}$. For dissolving unpolymerized poly- 

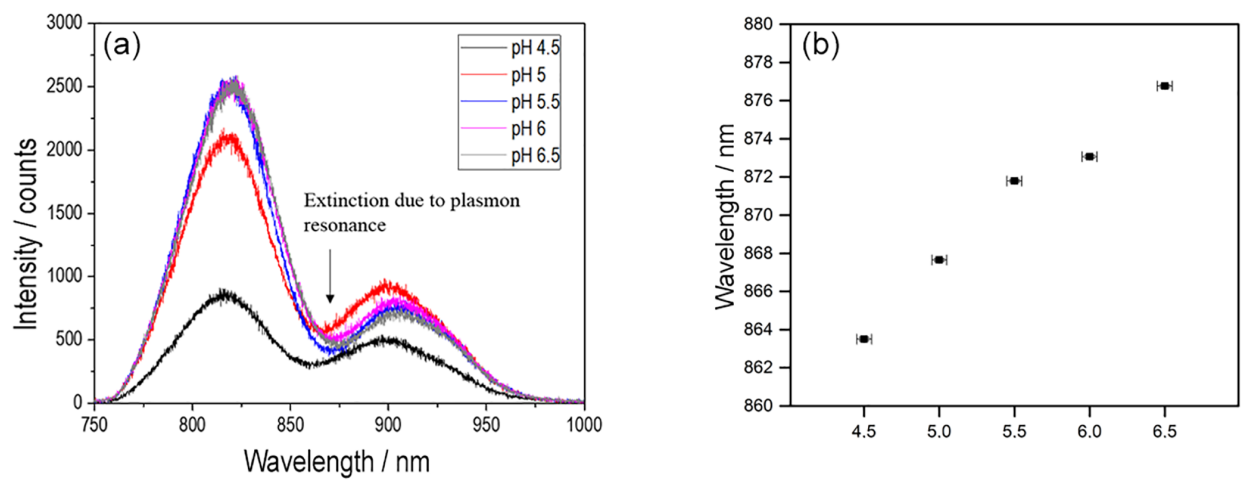

Figure 2. (a) Transmittance spectra and (b) plasmon resonance wavelengths for swelling states of the $\mathrm{pH}$-sensitive hydrogel for $\mathrm{pH}$ values between 4.5 and 6.5 .

mer chains, the hydrogels are purged with an ethanol-water solution for $24 \mathrm{~h}$. All chemicals were purchased from Sigma Aldrich (Taufkirchen, Germany).

\subsection{Sensor substrate}

The sensor substrates consist of a hexagonal $3 \times 3 \mathrm{~mm}^{2}$ nanopillar array (Fig. 1b), fabricated by means of nanoimprint lithography (NIL) (Yang et al., 2015) (in cooperation with GeSiM mbh, Großerkmannsdorf, Germany). A silicon master made by means of electron beam lithography is used to transfer the nanostructure into a PDMS layer. The PDMS then contains the negative of the master, which, in turn, is imprinted into an ultraviolet-sensitive SU-8 photoresist on glass using the $\mu$ ContactPrinter $4.1(\mathrm{GeSiM})$. Afterwards, the nanostructures are cured with ultraviolet radiation and the soft mold is removed. The nanopillars have a diameter of $230 \mathrm{~nm}$, a center-to-center distance of $450 \mathrm{~nm}$, and a height of $150 \mathrm{~nm}$. Finally, $2 \mathrm{~nm}$ of titanium and $25 \mathrm{~nm}$ of gold are sputtered on the nanopillars for adhesion and for surface plasmon activation, respectively.

\subsection{Surface functionalization}

The sensor substrates fabricated according to the protocol in Sect. 3.2 are cleaned for 10 min using isopropanol, highpurity water, and ethanol under ultrasonic conditions. After drying in a nitrogen stream, the substrates are inserted in an $\mathrm{O}_{2}$-plasma cleaner for $5 \mathrm{~min}$. For the surface functionalization the cleaned samples are placed in a beaker containing a $4 \mathrm{mM}$ (3-mercaptopropyl)trimethoxy silane solution, mixed with toluene, for $2.5 \mathrm{~h}$. With this surface functionalization, we predominantly intend to increase the lateral homogeneity of the hydrogel layer resulting from the spin-coating process (see Sect. 3.1). However, this functionalization to some extent also increases the adhesion of the hydrogel in comparison to a bare gold surface and therefore increases the durability of the sensor substrates.

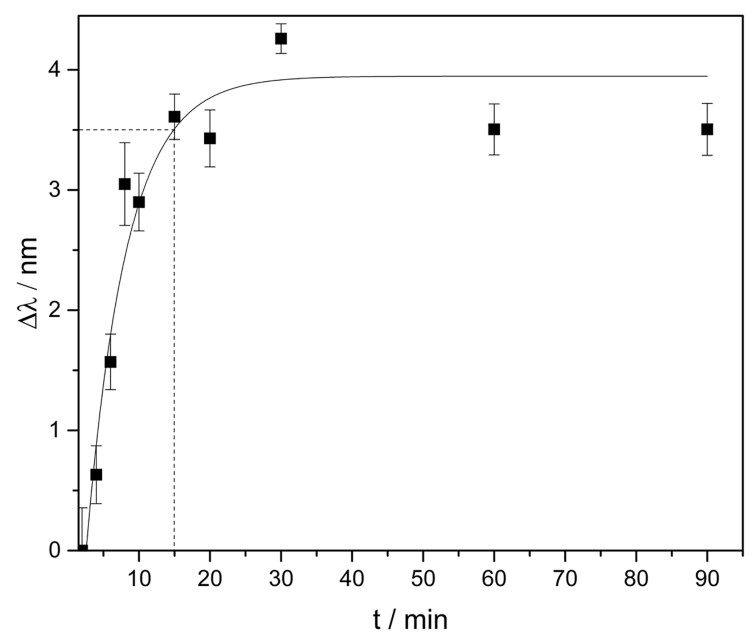

Figure 3. Change of the plasmon resonance wavelength induced by the hydrogel swelling in $\mathrm{pH} 4.5$ after a pre-swelling in $\mathrm{pH}$ 6.5. From the fit a response time of $15 \mathrm{~min}$ is derived after which the swelling reached $90 \%$ of the final steady-state value (indicated by the dashed line).

\section{Results \& discussion}

To attain different swelling degrees, the sensor substrate was placed in a petri dish containing liquids with different $\mathrm{pH}$ values for $15 \mathrm{~min}$. For every $\mathrm{pH}$ value the sensor substrate was taken out of the $\mathrm{pH}$ solution and a transmittance spectrum was measured with the setup described above. The superposition of the Gaussian LED excitation spectrum and the plasmonic extinction spectrum leads to a characteristic dip in the overall transmittance spectrum around $870 \mathrm{~nm}$ (see Fig. 2a). The spectral position of the transmittance dip represents the plasmon resonance wavelength of the gold nanostructure and depends on the refractive index of the hydrogel (Zalyubovskiy et al., 2012). This resonance wavelength was determined with a Gaussian fit for the transmittance spectra of every $\mathrm{pH}$ value. Figure 2 shows the transmittance spectra 
(a) and the resonance wavelength of these measurements for the $\mathrm{pH}$ values between 6.5 and 4.5 with intervals of 0.5 (b).

The spectra show a blueshift of the plasmon resonance wavelengths with decreasing $\mathrm{pH}$ value which corresponds to a decreasing refractive index of the hydrogel during swelling. The plotted uncertainty bars originate from the uncertainty of the $\mathrm{pH}$ electrode, which was used for the preparation of the $\mathrm{pH}$ solutions, and the standard deviations of the Gaussian fits for the resonance wavelengths. Figure $2 b$ indicates that the refractive index of the polymer decreases according to the raising content of water in the gel, which is in accordance with our expectation. The data show that the system is suitable for the detection of different $\mathrm{pH}$ values in aqueous liquids ranging from 4.5 to 6.5 .

In order to monitor the in situ swelling of the hydrogel and to determine the response time, we placed the sensor substrates in $\mathrm{pH} 6.5$ solution for $2 \mathrm{~h}$. Afterwards we observed the hydrogel swelling in $\mathrm{pH} 4.5$ over $90 \mathrm{~min}$. Figure 3 shows the change of the resonance wavelength induced by a swelling of the $\mathrm{pH}$-sensitive hydrogel over time.

The results show an exponential swelling of the hydrogel. After $15 \mathrm{~min}, 90 \%$ of the final steady-state value is reached, which shows an improvement of the response time by a factor of 2 in comparison to other systems exploiting this kind of hydrogel (Schmidt et al., 2016; Schulz et al., 2010). This improvement is caused by using a much thinner hydrogel layer $(30 \mu \mathrm{m}$ instead of $>100 \mu \mathrm{m})$, which, in turn, leads to lower diffusion times of the surrounding liquid into the gel and, hence, to a faster response to changing $\mathrm{pH}$. The layer thickness reported here represents the first step towards even shorter response times. For plasmonic readout, a hydrogel layer of around $1 \mu \mathrm{m}$ would be sufficient, which promises response times in the minute range or even below.

The error margins in Figs. 2 and 3 are still relatively large. We attribute this behavior to insufficient adhesion of the hydrogel on the substrate surface as there is no covalent binding between the hydrogel and the surface functionalization. To investigate adhesion, the substrates were placed in an ethanol-water solution for 5 days and compared to substrates on which we omitted the (3-mercaptopropyl)trimethoxy silane layer. The samples with surface functionalization show clearly better durability (flawless adhesion in the range of days instead of minutes for bare gold). However, for some of the functionalized substrates we still observe a delamination between hydrogel and the gold layer. Hence, although the current functionalization improves the adhesion it is still not strong enough to enable a stable sensor functionality. To avoid these effects, we are currently working on alternative functionalization protocols which will allow us to conduct more detailed signal stability tests.

\section{Conclusion and outlook}

In this paper we report on initial results concerning the optical detection of the swelling behavior of a $\mathrm{pH}$-sensitive hydrogel applicable in the physiological range. We applied a nanostructured gold surface supporting localized surface plasmons as an optical transducer for refractive index changes occurring during $\mathrm{pH}$-induced swelling of the hydrogel. The combination of a transducer and the HPMA/DMAEMA/TEGDMA/EG hydrogel composition is being studied for the first time. The results show a nearly linear blueshift of the plasmon resonance wavelength of the transmittance spectrum with increasing swelling of the hydrogel upon decreasing $\mathrm{pH}$ values. Furthermore, we could show an improvement of the response time of the sensor (by a factor of 2) in comparison to other approaches exploiting this specific hydrogel. Future work will focus on improving the adhesion between the gold and the hydrogel layer and on producing even thinner and patterned hydrogel layers.

Data availability. The data presented in this article are stored in an internal system according to the guidelines of the DFG.

Competing interests. The authors declare that they have no conflict of interest.

Acknowledgements. The authors thank GeSiM mbH, Großerkmannsdorf, Germany for the intense cooperation concerning plasmonic structure fabrication and Rüdiger Rosenkranz for the REM work. Funding from the DFG research training group 1865 and the BMBF VIP+ program under grant number 03VP01970 is gratefully acknowledged.

Edited by: Ulrich Schmid

Reviewed by: three anonymous referees

\section{References}

Gerlach, G., Guenther, M., Suchaneck, G., Sorber, J., Arndt, K.-F., and Richter, A.: Application of sensitive hydrogels in chemical and pH sensors, Macromol. Symp., 210, 403-410, https://doi.org/10.1002/masy.200450645, 2004.

Guenther, M., Gerlach, G., Wallmersperger, T., Avula, M. N., Cho, S. H., Xie, X., Devener, B. V., Solzbacher, F., Tathireddy, P., Magda, J. J., Scholz, C., Obeid, R., and Armstrong, T.: Smart Hydrogel-Based Biochemical Microsensor Array for Medical Diagnostics, Adv. Sci. Tech., 85, 47-52, https://doi.org/10.4028/www.scientific.net/AST.85.47, 2013.

Guenther, M., Wallmersperger, T., and Gerlach, G.: Piezoresistive Chemical Sensors Based on Functionalized Hydrogels, Chemosensors, 2, 145-170, https://doi.org/10.3390/chemosensors2020145, 2014.

Jiang, H., Markowski, J., and Sabarinathan, J.: Near-infrared optical response of thin film $\mathrm{pH}$ sensitive hydrogel coated 
on a gold nanocrescent array, Opt. Express, 17, 21802-7, https://doi.org/10.1364/OE.17.021802, 2009.

Kreibig, U. and Vollmer, M.: Optical Properties of Metal Clusters, Springer Series in Material Science, Springer-Verlag Berlin Heidelberg, https://doi.org/10.1007/978-3-662-09109-8, 1995.

Mack, N. H., Wackerly, J. W., Malyarchuk, V., Rogers, J. A., Moore, J. S., and Nuzzo, R. G.: Optical Transduction of Chemical Forces, Nano Lett., 7, 733-737, https://doi.org/10.1021/n10629759, 2007.

Piliarik, M., Šípová, H., Kvasnička, P., Galler, N., Krenn, J. R., and Homola, J.: High-resolution biosensor based on localized surface plasmons, Opt. Express, 20, 672-680, https://doi.org/10.1364/OE.20.000672, 2012.

Schmidt, U., Guenther, M., and Gerlach, G.: Biochemical piezoresistive sensors based on $\mathrm{pH}$ - and glucose-sensitive hydrogels for medical applications, De Gruyter, Curr. Dir. Biomed. Eng., 2, 117-121, https://doi.org/10.1515/cdbme-2016-0029, 2016.

Schulz, V., Gerlach, G., Günther, M., Magda, J. J., and Solzbacher, F.: Piezoresistive pH Microsensors Based on Stimuli-Sensitive Polyelectrolyte Hydrogels, Tech. Mess., 77, https://doi.org/10.1524/teme.2010.0045, 2010.

Tanaka, T. and Fillmore, D. J.: Kinetics of swelling of gels, J. Chem. Phys., 70, 1214, https://doi.org/10.1063/1.437602, 1979.

Tokarev, I., Tokareva, I., and Minko, S.: GoldNanoparticle-Enhanced Plasmonic Effects in a Responsive Polymer Gel, Adv. Materials, 20, 2730-2734, https://doi.org/10.1002/adma.200702885, 2008.
Wang, J. and Ward, M. D.: Piezoelectric pH Sensors: AT-Cut Quartz Resonators with Amphoteric Polymer Films, Anal. Chem., 65, 2553-2562, https://doi.org/10.1021/ac00067a002, 1993.

Wang, M., Yao, S., and Madou, M.: A long-term stable iridium oxide $\mathrm{pH}$ electrode, Sensor Actuat. B, 81, 313-315, https://doi.org/10.1016/s0925-4005(01)00972-8, 2002.

Wilhelm, D., Voigt, H., Treichel, W., Ferretti, R., and Prasad, S.: pH sensor based on differential measurements on one pH-FET chip, Sensor Actuat. B, 4, 145-149, https://doi.org/10.1016/09254005(91)80190-U, 1991.

Yang, S.-C., Hou, J.-L., Finn, A., Kumar, A., Ge, Y., and Fischer, W.-J.: Synthesis of multifunctional plasmonic nanopillar array using soft thermal nanoimprint lithography for highly sensitive refractive index sensing, Nanoscale, 7, 5760-5766, https://doi.org/10.1039/C5NR00472A, 2015.

Yao, J., Le, A., Gray, S. K., Moore, J. S., Rogers, J. A., and Nuzzo, R. G.: Functional Nanostructured Plasmonic Materials, Adv. Materials, 22, 1102-1110, https://doi.org/10.1002/adma.200904097, 2010.

Zalyubovskiy, S. J., Bogdanova, M., Deinega, A., Lozovik, Y., Pris, A. D., An, K. H., Hall, W. P., and Potyrailo, R. A.: Theoretical limit of localized surface plasmon resonance sensitivity to local refractive index change and its comparison to conventional surface plasmon resonance sensor, J. Opt. Soc. Am. A, 29, $994-$ 1002, https://doi.org/10.1364/JOSAA.29.000994, 2012. 